\title{
Fatores Associados à Satisfação com a Vida de Idosos Usuários de Unidades Básicas de Saúde
}

\author{
Associated Factors in Satisfaction with the Life of Elderly Users of Basic Health Units \\ Fatores Asociados a la Satisfacción con la Vida de los Ancianos Usuarios de Unidades \\ Básicas de Salud
}

\author{
Daniel Vicentini de Oliveira ${ }^{1}$ \\ Centro Universitário de Maringá \\ Anderson Rodrigues Brito \\ Centro Universitário Metropolitano de Maringá \\ Maria do Carmo Correia de Lima \\ Naelly Renata Saraiva Pivetta \\ Universidade Estadual de Campinas \\ José Roberto Andrade do Nascimento Júnior \\ Universidade Federal do Vale do São Francisco
}

\begin{abstract}
Resumo
Este estudo investigou os fatores associados à satisfação com a vida de 654 idosos usuários de Unidades Básicas de Saúde de Maringá, Paraná. Foi utilizado um questionário sociodemográfico, o International Physical Activity Questionnaire (IPAQ) e a Escala de Satisfação com a Vida. A análise dos dados foi realizada por meio dos testes de Kruskal-Wallis, Mann-Whitney e correlação de Spearman ( $p$ $<0,05)$. Verificou-se que os idosos com menor poder aquisitivo que se perceberam com saúde ruim e tomam medicamentos regularmente são menos satisfeitos com a vida do que seus pares. Os idosos fisicamente ativos se mostraram mais satisfeitos com a vida do que os sedentários/irregularmente ativos. A satisfação com a vida se associou com os dias de caminhada por semana $(r=0,15)$. Concluise que a renda familiar, a percepção de saúde, o uso de medicamentos e a atividade física são fatores intervenientes na satisfação com a vida.
\end{abstract}

Palavras-chave: velhice, saúde, promoção da saúde, satisfação com a vida

\begin{abstract}
This study investigated the associated factors involved in life satisfaction of 654 elderly users of Basic Health Units of Maringá, Paraná. A sociodemographic questionnaire was used, the International Physical Activity Questionnaire (IPAQ) and the Life Satisfaction Scale. Data analysis was performed using the Kruskal-Wallis, Mann-Whitney, and Spearman correlation tests $(p<0.05)$. Older people with lower purchasing power who were perceived with poor health and who take medications regularly are less satisfied with life than their peers $(p<0.05)$. The physically active elderly were more satisfied with life than the sedentary/irregularly active $(p=0.004)$. Satisfaction with life correlated with walking days per week $(r=0.15)$. It is concluded that family income, health perception, medication use, and physical activity are factors that intervene in life satisfaction.
\end{abstract}

Keywords: old age, health, health promotion, life satisfaction

\section{Resumen}

Este estudio investigó los factores asociados con la satisfacción con la vida de 654 ancianos usuarios de Unidades Básicas de Salud de Maringá, Paraná. Se utilizó un cuestionario sociodemográfico, el International Physical Activity Questionnaire (IPAQ) y la Escala de Satisfacción con la vida. El análisis de los datos fue realizado por medio de las pruebas de Kruskal-Wallis, Mann-Whitney y correlación de Spearman $(p<0,05)$. Se verificó que los ancianos con menor poder adquisitivo que se percibieron con mala salud y que toman medicamentos regularmente son menos satisfechos con la vida que sus pares. Los ancianos físicamente activos se mostraron más satisfechos con la vida que los sedentarios/ irregularmente activos. La satisfacción con la vida se asoció con los días de caminata por semana

\footnotetext{
${ }^{1}$ Endereço de contato: Avenida Guedner, 1610, Jardim Aclimação, Maringá, Paraná, CEP 87050-900. E-mail: d.vicentini@hotmail.com
} 
$(r=0,15)$. Se concluye que la renta familiar, la percepción de salud, el uso de medicamentos y la actividad física son factores intervinientes en la satisfacción con la vida.

Palabras clave: vejez, salud, promoción de la salud, satisfacción con la vida

\section{Introdução}

No Brasil, o aumento da população idosa representa um desafio para o desenvolvimento de programas e ações intersetoriais que visam garantir um olhar integral e amplo para proporcionar uma vida com dignidade, qualidade e satisfação pessoal para os idosos (Camarano, \& Pasinato, 2004; Joia, \& Ruiz, 2013).

O envelhecimento é um processo natural e dinâmico (Mazo, Loposcki, Ananda, \& Prevê, 2007). É determinado por fatores intrínsecos e extrínsecos (Lima, \& Delgado, 2017). Os fatores intrínsecos contemplam as alterações biológicas, cognitivas e fisiológicas naturais do organismo que culminam na diminuição da sua capacidade de adaptação e regeneração diante de estímulos estressores (Mazo et al., 2007). Os fatores extrínsecos também influenciam nesse processo, podendo atenuar ou acentuar as alterações advindas do envelhecimento, entre os quais podemos destacar o estilo de vida, acesso à saúde, condição social, econômica e ambiental (Lima, \& Delgado, 2017).

Assim, nota-se que o envelhecimento é algo bastante singular para cada indivíduo (Lima, \& Delgado, 2017). Da mesma forma, de modo individual, cada idoso percebe a sua vida e se mostra satisfeito ou não com ela, levando em consideração sua condição de saúde atual e também suas expectativas pessoais (Mantovani, Lucca, \& Neri, 2016; Neri, \& Pinto, 2013).

A satisfação com a vida é baseada na subjetividade e está relacionada a experiências e anseios pessoais, o que a torna objeto complexo e de difícil mensuração. Cada indivíduo avalia a satisfação com a vida com base na comparação entre as circunstâncias de sua própria vida e padrões ideais estabelecidos por ele. Saúde, trabalho, condições de moradia, relações sociais e autonomia estão entre os principais domínios considerados na medida particular de satisfação com a vida. Pode-se dizer que a satisfação com a vida retrata o bem-estar subjetivo pessoal e a motivação que leva as pessoas a viverem suas experiências de vida de maneira positiva (Joia, Ruiz, \& Donalisio, 2007).

Nesse contexto, mensurar a satisfação com a vida e estudar os fatores que nela interferem é uma ferramenta que tem sido usada para melhor compreender a população idosa (Mantovani et al., 2016; Pinto, \& Neri, 2013).

Estudos demonstram que a satisfação com a vida é influenciada por sexo, idade, condição social e econômica, assim como pela cultura e ambiente, e que é um forte indicador de qualidade de vida percebida (Pinto, \& Neri, 2013; Sposito, D’Elboux, Neri, \& Guariento, 2013; Tomomitsu, Valente, Perracini, \& Liberalesso Neri, 2016; Mantovani et al., 2016). Pinto e Neri (2013) demonstraram que idosos de baixa renda apresentaram menor pontuação em domínios de satisfação; Sposito et al. (2013) demonstraram que a idade elevada se correlacionou com a maior satisfação com a vida. Tomomitsu et al. (2016) evidenciaram que idosos com bom nível de suporte social demonstraram maior nível de satisfação com a vida.

Além disso, a satisfação com a vida pode ser considerada um guia norteador para instituições que integram as redes de suporte social, como é o caso da Unidade Básica de Saúde (UBS) (Hsu, 2012). 
A UBS é uma rede de apoio social formal, cujo papel é proporcionar à população e ao idoso prevenção e manejo de doenças, assistência médica e psicológica (Alvarenga, 2011 Oliveira, Liberalesso Neri, \& D’Elboux, 2016). Para os idosos, a UBS é essencial, pois nesta etapa da vida se tornam mais passíveis de adoecer, de apresentar enfermidades crônicas, degenerativas, quedas, lesões, declínio cognitivo e incapacidade funcional (Boeckxstaens et al., 2015). Portanto contar com uma rede de suporte social que considere suas necessidades e expectativas é primordial e, para isso, a mensuração da satisfação com a vida é uma ferramenta altamente capaz de atender a esta diligência.

Posto isto, esta pesquisa teve como objetivo investigar os fatores intervenientes na satisfação com a vida de idosos usuários de UBS.

\section{Métodos}

Trata-se de um estudo epidemiológico de corte observacional e transversal, aprovado pelo Comitê de Ética em Pesquisa do Centro Universitário de Maringá (UNICESUMAR), por meio do parecer número 1.777.797/2016.

\section{Participantes}

Segundo dados obtidos junto à Secretária de Saúde do município de Maringá, Paraná (PR), a população alvo é composta de 42.258 idosos (2016). Foram utilizados dois tipos de amostragens: randômica, para a seleção das UBS, e não randômica, por cotas para a seleção dos idosos. A amostra inicial a ser considerada foi de 595 idosos, adicionando 10\% de possíveis perdas. A amostra final foi composta com 654 idosos, com média de idade de 69,0 anos (DP $=7,5)$, de ambos os sexos, considerando um nível de confiança de 95\% e 4\% de margem de erro. O software utilizado para obtenção dos cálculos foi o StatDisk versão 8.4.

A amostra foi composta com idosos frequentadores das Unidades Básicas de Saúde (UBS) do município. As UBS das quais fazem parte os idosos foram subdivididas em quatro regiões: leste (7 UBS), que contempla $21,8 \%$ da população; norte (8 UBS), com 34,5\% da população; oeste ( 8 UBS), com 23,2\% da população; e sul ( 8 UBS), que contempla 20,4\% da população total de idosos da cidade. Conhecendo a composição das regiões, foram selecionadas por sorteio três UBS para serem avaliadas em cada uma dessas regiões.

Após definido o tamanho da amostra em cada região e selecionada a UBS, foi importante manter a proporção de idosos da população na amostra; sendo assim, os cálculos para obtenção da amostra final por UBS de acordo com sexo foram proporcionais aos populacionais.

Foram incluídos no estudo idosos de ambos os sexos com idade igual ou superior a 60 anos, com capacidade de fala e audição preservadas, que permitam a aplicação dos questionários. O Mini Exame do Estado Mental (MEEM) foi utilizado para excluir idosos com deficits cognitivos importantes. As notas de corte utilizadas para exclusão pelo MEEM foram: 17 para os analfabetos; 22 para idosos com escolaridade entre 1 e 4 anos; 24 para os com escolaridade entre 5 e 8 anos; e 26 aos que tiverem 9 anos ou mais anos de escolaridade (Folstein, Folstein, \& McHugh, 1975; Brucki, Nitrini, Caramelli, Bertolucci, \& Okamoto, 2003). 


\section{Instrumentos}

Para a caracterização dos idosos, foi utilizado um questionário semiestruturado, composto de informações referentes à idade (60 a 69 anos; 70 a 79 anos; 80 a 89 anos; 90 anos ou mais), sexo (masculino; feminino), estado civil (casado ou vive com o companheiro; solteiro; divorciado, casado ou dequisitado; viúvo), raça (caucasiana; negra; oriental), situação ocupacional (trabalha ou não trabalha para obter renda própria), renda mensal em salário mínimo (SM) de referência no Censo Demográfico 2016 do Instituto Brasileiro de Geografia e Estatística (IBGE) (1 a 2 SM, 2,1 a 3 SM, > 3 SM), aposentadoria (sim ou não), escolaridade (não estudou; Ensino Fundamental incompleto, Ensino Fundamental completo, Ensino Médio incompleto, Ensino Médio completo, Ensino Superior), autopercepção do estado de saúde (ruim, regular, bom, muito bom), histórico de quedas nos últimos seis meses (sim, não; e quantidade de quedas), quantidade de medicamentos utilizados (1, 2, 3 ou mais).

O nível de atividade física dos idosos foi avaliado utilizando-se a versão curta do Questionário Internacional de Atividade Física (IPAQ). Este instrumento avalia atividades físicas realizadas no tempo de lazer, como deslocamento de um lugar ao outro, serviços domésticos e atividades ocupacionais (Matsudo et al., 2001). Foi considerado o tempo de 150 minutos semanais de atividades físicas para o sujeito ser classificado como fisicamente ativo; com menos de dez minutos semanais, o sujeito foi considerado sedentário; e aqueles que realizavam ao menos dez minutos, mas não alcançavam os 150 minutos, foram considerados insuficientemente ativos.

A Escala de Satisfação com a Vida (SV) é um instrumento proposto por Diener e colaboradores (Diener, Emmons, Larsen, \& Griffin, 1985), composta de cinco itens, sendo as respostas graduadas de acordo com uma escala tipo Likert, que vai de $1=$ discordo totalmente a 7 = concordo totalmente. Essa escala tem o propósito de avaliar o julgamento que as pessoas fazem acerca da sua própria SV, sendo elas próprias a eleger, de acordo com seus valores e interesses, os aspectos a serem considerados para expressar tal satisfação. De um possível escore total de 35 pontos, quanto mais próximo dele, melhor a SV do idoso.

\section{Coleta de dados}

Os dados foram coletados em 12 UBS, divididas nas quatro regiões (norte, sul, leste e oeste), das 33 UBS do município de Maringá, selecionadas por meio de sorteio, após autorização da CECAPS. A coleta foi realizada por uma equipe de pesquisadores, que foram devidamente treinados para tal fim. Um piloto com 30 idosos foi realizado previamente ao estudo.

Os idosos voluntários foram abordados pelo pesquisador responsável ou pela equipe de pesquisadores, informados quanto à justificativa, objetivos e procedimentos a serem realizados, conforme orientações para pesquisa com seres humanos constantes na Resolução 196/96 do Conselho Nacional de Saúde. Após esses procedimentos, aqueles que aceitaram participar da pesquisa assinaram o Termo de Consentimento Livre e Esclarecido (TCLE). Optou-se pela entrevista direta na aplicação dos questionários, em razão da possível dificuldade de leitura, problemas visuais e de compreensão dos questionamentos. 


\section{Análise dos dados}

A análise dos dados foi realizada por meio do Software SPSS 22.0, mediante uma abordagem de estatística descritiva e inferencial. Foi utilizada frequência absoluta e relativa como medida descritiva para as variáveis categóricas. Inicialmente, foi verificada a normalidade da distribuição de dados por meio do teste Kolmogorov-Smirnov. Como os dados não apresentaram distribuição normal, foram utilizadas Mediana (Md) e Quartis (Q1; Q3) para a caracterização dos resultados. Na comparação do grau de satisfação com a vida em função das variáveis sociodemográficas e das condições de saúde, foi utilizado o teste de Kruskal-Wallis e o "U" de Mann-Whitney. A correlação entre a satisfação com a vida e o nível de atividade física foi verificada por meio do coeficiente de Spearman. Considerou-se um nível de significância de $p<0,05$.

\section{Resultados}

Nota-se a prevalência de idosos do sexo feminino (56,0\%), casados (61,3\%), com idade entre 60 e 69 anos (59,2\%), renda mensal de 1 a 2 SM (70,0\%), da raça branca $(81,0 \%)$ e aposentados (75,0\%). Observou-se também que 43,0\% dos idosos têm Ensino Fundamental incompleto.

Já em relação ao perfil de saúde dos idosos usuários das UBS do município de Maringá, verificou-se a prevalência de idosos com percepção de saúde boa (48,5\%), que tomam de 1 a 2 medicamentos regularmente $(43,9 \%)$, não tiveram histórico de quedas $(83,7 \%)$ ou quase quedas nos últimos seis meses (79,7\%) e com nível de atividade física ativo/muito ativo (60,7\%).

Em relação à satisfação com a vida, os idosos obtiveram mediana de 27,0. Ao comparar o grau de satisfação com a vida dos idosos usuários das UBS do município de Maringá em função das variáveis sociodemográficas (Tabela 1), verificou-se diferença significativa no grau de satisfação com a vida apenas para a renda mensal $(p=0,015)$, indicando que os idosos que recebem de um a dois SM são menos satisfeitos com a vida do que os idosos com maior poder aquisitivo (mais de $3 \mathrm{SM}$ ); não houve diferença significativa no grau de satisfação com a vida em função do sexo, faixa etária, estado civil e aposentadoria.

Tabela 1

Comparação do grau de satisfação com a vida dos idosos usuários das UBS do município de Maringá, $P R$, em função das variáveis sociodemográficas

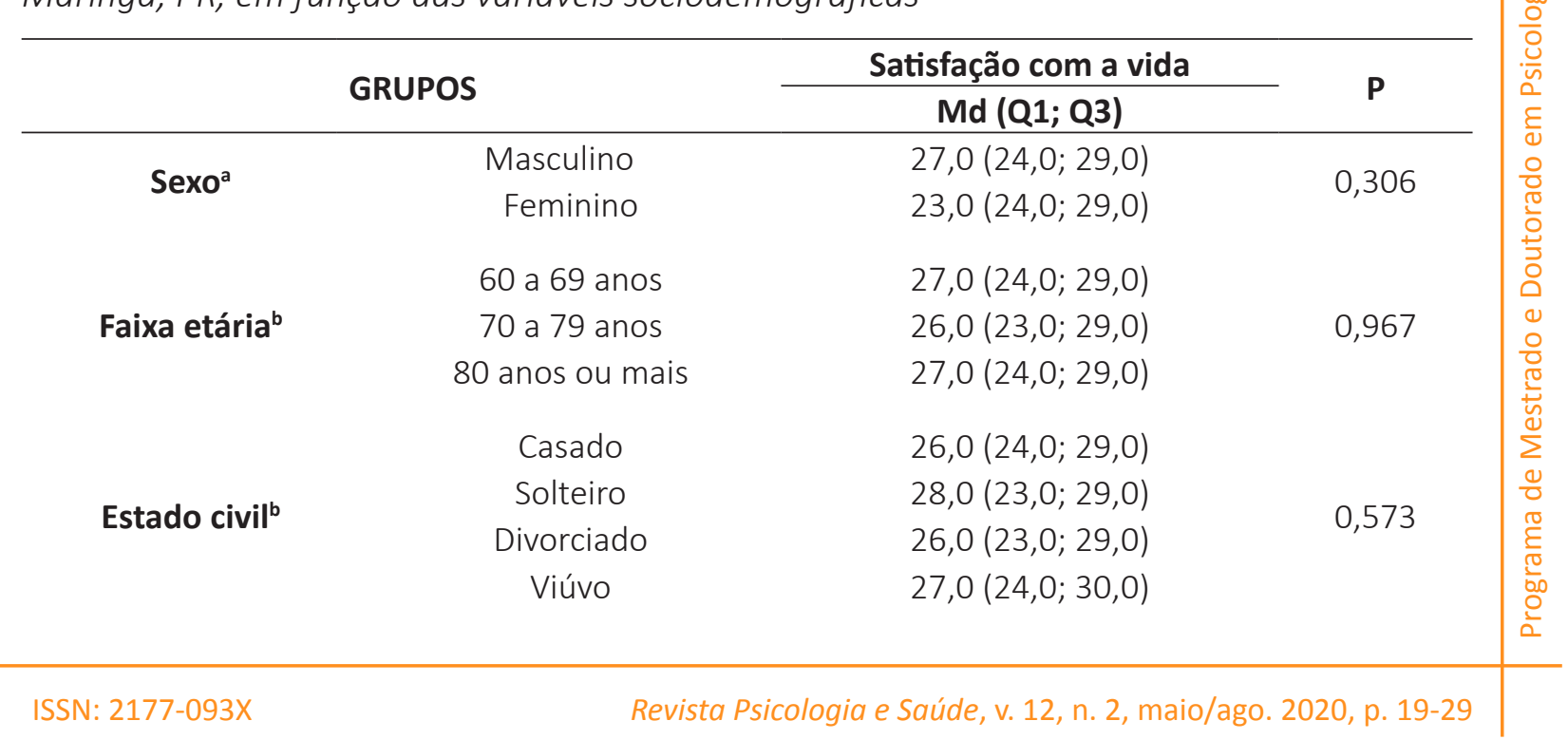




\begin{tabular}{|c|c|c|c|}
\hline \multirow{2}{*}{\multicolumn{2}{|c|}{ GRUPOS }} & \multirow{2}{*}{$\begin{array}{c}\text { Satisfação com a vida } \\
\text { Md (Q1; Q3) }\end{array}$} & \multirow{2}{*}{$\mathbf{P}$} \\
\hline & & & \\
\hline \multirow{3}{*}{ Renda mensal $^{b}$} & 1 a 2 SM & $26,0(23,0 ; 29,0)$ & \multirow{3}{*}{$0,015^{*}$} \\
\hline & 2,1 a $3 \mathrm{SM}$ & $28,0(24,3 ; 30,0)$ & \\
\hline & Mais de 3 SM & $28,0(25,0 ; 30,0)^{\dagger}$ & \\
\hline \multirow{2}{*}{ Aposentadoria ${ }^{a}$} & Sim & $27,0(24,0 ; 29,0)$ & \multirow{2}{*}{0,637} \\
\hline & Não & $27,0(23,0 ; 29,0)$ & \\
\hline
\end{tabular}

Ao comparar o grau de satisfação com a vida dos idosos usuários das UBS do município de Maringá em função das condições de saúde (Tabela 2), verificou-se diferença significativa para a percepção de saúde $(p=0,001)$, uso de medicamentos $(p=0,048)$ e nível de atividade física $(p=0,004)$. Esses resultados indicam que os idosos com percepção de saúde ruim são menos satisfeitos com a vida do que os idosos com percepção de saúde boa e regular, e que os idosos que tomam regularmente mais de 2 medicamentos são menos satisfeitos com a vida do que os idosos que não tomam nenhum medicamento. Além disso, os idosos com nível de atividade física muito ativo/ativo são mais satisfeitos com a vida do que os idosos irregularmente ativos e sedentários. Não houve diferença significativa no grau de satisfação com a vida em função do histórico de quedas ( $p=0,300)$.

\section{Tabela 2}

Comparação do grau de satisfação com a vida dos idosos usuários das UBS do município de Maringá, PR, em função das condições de saúde

\begin{tabular}{|c|c|c|c|}
\hline \multirow{2}{*}{\multicolumn{2}{|c|}{ GRUPOS }} & \multirow{3}{*}{$\begin{array}{c}\text { Satisfação com a vida } \\
\text { Md (Q1;Q3) } \\
27,0(25,0 ; 30,0)^{\dagger}\end{array}$} & \multirow{2}{*}{$\mathbf{P}$} \\
\hline & & & \\
\hline \multirow{4}{*}{ Percepção de saúde ${ }^{b}$} & Boa & & \\
\hline & Regular & $26,5(24,0 ; 29,0)$ & $0,001 *$ \\
\hline & Ruim & $23,0(16,0 ; 28,0)$ & \\
\hline & Nenhum & $28,0(24,0 ; 30,0)^{\dagger}$ & \\
\hline \multirow[t]{3}{*}{ Medicamentos $^{\mathrm{b}}$} & 1 a 2 & $27,0(24,0 ; 29,0)$ & $0,048^{*}$ \\
\hline & Mais de 2 & $26,0(23,0 ; 29,0)$ & \\
\hline & Muito ativo/ativo & $27,0(24,0 ; 30,0)^{\dagger}$ & \\
\hline \multirow{2}{*}{ Nível de atividade físicab } & Irregularmente ativo & $26,0(23,0 ; 29,0)$ & $0,004^{*}$ \\
\hline & Sedentário & $26,0(23,0 ; 29,0)$ & \\
\hline \multirow{2}{*}{$\begin{array}{l}\text { Quedas nos últimos seis } \\
\text { meses }^{\mathrm{a}}\end{array}$} & Sim & $26,0(23,0 ; 29,0)$ & \multirow{2}{*}{0,300} \\
\hline & Não & $27,0(24,0 ; 29,0)$ & \\
\hline \multicolumn{4}{|c|}{$\begin{array}{l}\text { *Diferença significativa - } p<0,05 \text { (Teste "U" de Mann-Whitneya e Kruskal-Wallisb: †Ruim com Boa e Regular; }+ \\
\text { Mais de } 2 \text { com Nenhum; † Muito ativo/ativo com Irregularmente ativo e Sedentário). }\end{array}$} \\
\hline \multicolumn{4}{|c|}{$\begin{array}{l}\text { Na Tabela 3, verificou-se que a satisfação com a vida se correlacionou significativamente } \\
(p<0,05) \text { com os dias de caminhada por semana }(r=0,15) \text {, indicando que a prática da ca- } \\
\text { minhada tem uma relação diretamente proporcional à satisfação com a vida, entretanto tal } \\
\text { relação é fraca. }\end{array}$} \\
\hline ISSN: 2177-093X & Revista Psi & aúde, v. 12, n. 2, maio/a & 20 , p. $19-29$ \\
\hline
\end{tabular}


Tabela 3

Correlação entre o nível de atividade física e a ansiedade dos idosos usuários das UBS do município de Maringá, $P R$

\begin{tabular}{|c|c|c|c|c|c|c|c|c|c|c|}
\hline \multirow[t]{2}{*}{ VARIÁVEIS } & \multicolumn{9}{|c|}{ Nível de atividade física } & \multirow{2}{*}{$\begin{array}{c}\begin{array}{c}\text { Sat. } \\
\text { com a } \\
\text { vida }\end{array} \\
10 \\
\end{array}$} \\
\hline & 1 & 2 & 3 & 4 & 5 & 6 & 7 & 8 & 9 & \\
\hline 1. Dias de caminhada & & $0,56 *$ & $0,77 *$ & $0,23^{*}$ & $0,14 *$ & $0,18 *$ & $-0,06$ & $-0,08$ & $-0,07$ & $0,15 *$ \\
\hline 2. Min. caminhada $\mathrm{p} / \mathrm{dia}$ & & & $0,89 *$ & $0,26^{*}$ & $0,31^{*}$ & $0,28 *$ & 0,02 & 0,01 & 0,01 & 0,06 \\
\hline 3. Min. caminhada $\mathrm{p} / \mathrm{sem}$. & & & & $0,26^{*}$ & $0,26 *$ & $0,30 *$ & 0,02 & 0,01 & 0,01 & 0,09 \\
\hline 4. Dias de ativ. mod. & & & & & $0,86 *$ & $0,93 *$ & $0,47 *$ & $0,47 *$ & $0,47^{*}$ & 0,07 \\
\hline 5. Min. ativ. mod. $\mathrm{p} / \mathrm{dia}$ & & & & & & $0,94^{*}$ & $0,50 *$ & $0,53 *$ & $0,53^{*}$ & 0,01 \\
\hline 6. Min. ativ. mod. p/ sem. & & & & & & & $0,51^{*}$ & $0,53^{*}$ & $0,53^{*}$ & 0,04 \\
\hline 7. Dias de ativ. vigorosa & & & & & & & & $0,98 *$ & $0,98 *$ & $-0,07$ \\
\hline 8. Min. ativ. vig. p/ dia & & & & & & & & & $0,99 *$ & $-0,08$ \\
\hline $\begin{array}{l}\text { 9. Min. ativ. vig. p/ sem. } \\
\text { 10. Satisfação com a vida }\end{array}$ & & & & & & & & & & $-0,07$ \\
\hline
\end{tabular}

*Correlação significativa $-p<0,05$. Nota: 1. Dias de caminhada; 2. Min. caminhada p/ dia; 3. Min. caminhada p/ sem.; 4. Dias ativ. moderada; 5. Min. ativ. mod. p/ dia; 6. Min. ativ. mod. p/ sem; 7. Dias de ativ. vigorosa; 8. Min. ativ. vigorosa p/ dia; 9. Min. ativ. vig. p/ sem.; 10. Satisfação com a vida.

\section{Discussão}

No presente estudo, os dados sociodemográficos de maioria do sexo feminino, casada, tendo como a cor de referência a branca e ganhando até dois SM seguem as referências do Censo Demográfico do Instituto Brasileiro de Geografia e Estatística (2016) e dos estudos de Pilger, Menon e Mathias (2011) e Drummond e Alves (2013), que apresentaram dados bem próximos.

Em relação à satisfação com a vida, os idosos obtiveram mediana de 27,0, indicando moderada SV. A maioria dos idosos com boa satisfação com a vida apresentou renda mensal de até dois SM. A renda mensal de até dois SM é uma característica comum em indivíduos nessa faixa etária pelo fato de eles serem, na maioria das vezes, aposentados. Além disso, essa condição econômica reflete a ocupação que o idoso exercia antes da aposentadoria e também o baixo grau de instrução. No estudo de Santos, Santana, Santos, Silva e Santos (2014), a ocupação mais incidente foi de aposentados e com renda mensal de até 2 SM, corroborando os dados desta pesquisa. Porém os resultados da presente pesquisa destoam do estudo realizado por Mantovani et al. (2016), no qual, ao avaliarem a SV, o quesito saúde foi o mais relevante e o item "recursos financeiros" foi o menos citado, o que obteve menor relevância para os idosos avaliados, apesar de os autores reconhecerem que é um fator que está fortemente associado à velhice bem-sucedida (Mantovani et al., 2016). Essa diferença observada pode estar relacionada à diferença de idade entre os idosos dos dois estudos. Apesar de ambos estarem voltados para a população idosa, a pesquisa de Mantovani et al. (2016) incluiu idosos de 65 a 80 anos e mais, com idade média de 72 anos. Já o presente estudo incluiu idosos a partir de 60 anos e a idade variou até 80 anos e mais, porém houve o predomínio de participantes na faixa etária de 60 a 69 anos. Embora a diferença seja pequena, sabe-se que, com o avançar da idade, a saúde declina e, portanto, passa a ter uma 
importância ainda maior para o idoso, que começa a experimentar as consequências do declínio na saúde, como doenças crônico degenerativas, quedas, dor, entre outras, ao contrário do idoso comparativamente mais jovem. Replicando o achado de Mantovani et al. (2016), Sposito et al. (2013), ao investigarem a associação entre SV e idade, apontaram que os idosos mais velhos apresentaram melhores pontuações na satisfação com vida, mesmo diante do declínio funcional, por exemplo.

Em relação às condições de saúde, grande parte dos entrevistados referiu ter uma boa saúde. Embora Alves e Rodrigues (2005) afirmem que os idosos podem superestimar a sua condição de saúde para mostrar autossuficiência, por medo de institucionalização ou de precisar de cuidados, o presente estudo apresenta dados consistentes para validar que os idosos realmente apresentam boa percepção de saúde: o baixo histórico de quedas e quase quedas nos últimos seis meses. A queda é um marcador funcional importante, que reflete desfechos adversos de saúde, tais como declínio de saúde, deficit cognitivo, redução da acuidade visual, polifarmácia, uso de medicamentos psicoativos, redução da capacidade física, tontura, dor, diabetes, artrite, depressão, fragilidade, risco de institucionalização e morte (Perracini, \& Ramos, 2002; Soares, Moraes, Ferriolli, \& Perracini, 2014). Diante destas informações, depreende-se que de diversas maneiras o estado de saúde dos idosos avaliados encontra-se preservado.

De acordo com Confortin et al. (2017), o nível de autonomia e autossuficiência está relacionado a uma melhor percepção de saúde. Assim, quanto menos doenças e medicação associada, melhor é a percepção de saúde em idosos, sendo um dos fatores para melhor satisfação com a vida. No presente estudo, foi verificado que os idosos com percepção de saúde ruim e que tomam regularmente mais de dois medicamentos são menos satisfeitos com a vida do que os idosos com percepção de saúde boa e regular e que não tomam nenhum medicamento.

No que se refere ao nível de atividade física e SV, os idosos do presente estudo com nível de atividade física muito ativo/ativo são mais satisfeitos com a vida do que os idosos irregularmente ativos e sedentários. De acordo com Silva, Teixeira, Soares, \& Alves (2016), quanto mais ativo o sujeito se encontra, melhor será a qualidade de vida na velhice. Em contrapartida, nos resultados apresentados pelo estudo proposto, pouco se pôde observar essa correlação, pelo fato de o nível de ansiedade estar correlacionado significantemente, e em relação à caminhada esses dados não foram satisfatórios.

Para Joia e Ruiz (2013), a determinação do grau de satisfação com a vida em geral entre idosos está relacionada à sensação de conforto e bem-estar, independentemente de serem indicadores de renda ou de estrato social.

Consideramos que novos estudos se fazem necessários com o intuito de relacionar bem-estar e SV na velhice e, a partir disso, medidas de intervenção podem ser desenvolvidas. A SV é um constructo complexo e subjetivo, no qual parâmetros pessoais estão envolvidos, portanto, antes de transpor essa medida para o cotidiano da prática de saúde pública, estudos mais sensíveis e com um espaço amostral amplo são necessários.

O que o presente estudo permitiu concluir é que a renda igual ou superior a $3 \mathrm{SM}$, percepção de saúde boa e regular, não fazer uso diário de medicamentos e ser fisicamente ativo são variáveis que se associam positivamente à SV. 
Estudos futuros podem ampliar seus resultados usando instrumentos capazes de quantificar a satisfação dos idosos com relação ao suporte social percebido, ao atendimento em saúde ofertado pela UBS que frequenta, ao acesso a medicamentos de alto custo e pelo desconhecimento das atividades físicas praticadas, tendo em vista que este pode ser um fator protetor para a preservação da saúde de idosos. Como limitações do estudo, podem ser mencionados os seguintes itens não explorados: arranjo familiar, investigação de sintomas depressivos e rastreio de doenças crônicas e religiosidade, que são dados que contribuem para a comparação com outros estudos da área.

\section{Referências}

Alvarenga, M. R. M. (2011). Social support networks for elderly patients attended by Family Health teams. Ciência \& Saúde Coletiva, 16(5), 2603-2611.

Alves, L. S., \& Rodrigues, R. N. (2005). Determinantes da autopercepção de saúde entre idosos do Município de São Paulo, Brasil. Revista Panamericana de Salud Publica, 17(5/6), 333-341.

Boeckxstaens, P., Vaes, B., Legrand, D., Dalleur, O., De Sutter, A., \& Degryse J. M., (2015). The relationship of multimorbidity with disability and frailty in the oldest patients: $A$ cross-sectional analysis of three measures of multimorbidity in the BELFRAIL cohort. The European journal of general practice, 21(1), 39-44.

Brucki, S. M. D., Nitrini, R., Caramelli, P., Bertolucci, P. H. F., \& Okamoto, I. H. (2003). Sugestões para o uso do mini exame do estado mental no Brasil. Arquivos de Neuropsiquiatria, 61(3B), 777-781.

Camarano, A. A. \& Pasinato, M. T. (2004). O envelhecimento populacional na agenda das políticas públicas. In, A. A. Camarano (Org.), Os novos idosos brasileiros: Muito além dos 60? Rio de Janeiro: IPEA.

Confortin, S. C., Schneider, I. J. C., Antes, D. L., Cembranel, F., Ono, L. M., Marques, L. . . D’Orsi, E. (2017). Condições de vida e saúde de idosos: resultados do estudo de coorte EpiFloripa Idoso. Epidemiologia e Serviços de Saúde, 26(2), 305-317.

Diener, E., Emmons, R. A., Larsen, R. J., \& Griffin, S. (1985). The satisfaction with life scale. Journal of Personality Assessment, 49, 71-75.

Drummond, A., \& Alves, E. D. (2013). Perfil socioeconômico e demográfico e a capacidade funcional de idosos atendidos pela Estratégia Saúde da Família de Paranoá, Distrito Federal. Revista Brasileira de Geriatria e Gerontologia, 16(4), 727-738.

Folstein, M. F., Folstein, S. E., \& McHugh, P. R. (1975). Mini Mental state - A practical method for grading the cognitive state of patients for the clinician. Journal of Psychiatric research, 12,189-198.

Hsu, H. C. (2012). Trajectories and covariates of life satisfaction among older adults in Taiwan. Archives of Gerontology and Geriatrics, 55(1), 210-216.

Instituto Brasileiro de Geografia e Estatística (IBGE). (2016). Censo demográfico IBGE. Disponível em http://www.ibge.gov.br.

Joia, L. C., Ruiz, T., \& Donalisio, M. R. (2007). Condições associadas ao grau de satisfação com a vida entre a população de idosos. Revista de Saúde Pública, 41(1), 131-138.

Joia, L. C., \& Ruiz, T. (2013). Satisfação com a vida na percepção dos idosos. Revista Kairós Gerontologia, 16(6), 79-102. 
Lima, A. P., \& Delgado, E. I. (2017). A melhor idade do Brasil: aspectos biopsicossociais decorrentes do processo de envelhecimento. ACTA Brasileira do Movimento Humano, 1(2).

Mantovani, E. P., Lucca, S. R., \& Neri, A. N. (2016). Associações entre significados de velhice e bem-estar subjetivo indicado por satisfação em idosos. Revista Brasileira de Geriatria e Gerontologia 19(2), 203-222.

Matsudo, S., Araújo, T., Matsudo, V., Andrade, D., Andrade, E., Oliveira, L. C., \& Braggion, G. (2001). Questionário Internacional de Atividade Física (Ipaq): Estudo de Validade e reprodutibilidade no Brasil. Revista Brasileira de Atividade Física \& Saúde, 6(2), 5-18.

Mazo, G., Liposcki, D., Ananda, C., \& Prevê, D. (2007). Condições de saúde, incidência de quedas e nível de atividade física dos idosos. Revista Brasileira de Fisioterapia, 11(6), 437-442.

Oliveira, D. C., Liberalesso Neri, A., \& D’Elboux, M. J. (2016). Ausência de expectativa de suporte para o cuidado aos idosos da comunidade. Revista Brasileira de Enfermagem, 69(3).

Perracini, M. R., \& Ramos, L. R. (2002). Fatores associados a quedas em uma coorte de idosos residentes na comunidade. Revista de Saúde Pública, 36(6), 709-716.

Pilger, C., Menon, M. H., \& Mathias, T. A. F. (2011). Características sociodemográficas e de saúde de idosos: Contribuições para os serviços de saúde. Revista. Latino-Americana de Enfermagem, 19(5),1-9.

Pinto, J. M., \& Neri, A. L. (2013). Doenças crônicas, capacidade funcional, envolvimento social e satisfação em idosos comunitários: Estudo Fibra. Ciência \& Saúde Coletiva, 18(12), 3449-3460.

Santos, A. D., Santana, W. B., Santos, A. M. D., Silva, G. M., \& Santos, M. B. (2014). Avaliação de risco cardiovascular em idosos segundo os critérios de Framingham. Scientia Plena, 10(10), 1-10.

Silva, P. L. N., Teixeira, C. I. B. F., Soares, E. D., \& Alves, C. R. (2016). Avaliação da qualidade de vida de idosos praticantes de atividade física de uma unidade básica de saúde de Minas Gerais. Revista da Universidade Vale do Rio Verde,14(2), 24-35.

Soares, W. J. S., Moraes, S. A., Ferriolli, E. \& Perracini, M. R.(2014). Fatores associados a quedas e quedas recorrentes em idosos: estudo de base populacional. Revista Brasileira de Geriatria e Gerontologia, 17(1), 49-60.

Souza, W. C., Mascarenhas, L. P. G., Grzelczak, M. T., Tajes Junior, D., Brasilino, F. F., \& Lima, V. A. de.. Exercício físico na promoção da saúde na terceira idade. (2015). Saúde e Meio Ambiente: Revista Interdisciplinar, 4(1), 55-65.

Sposito, G., D’Elboux, M. J., Neri, A. L. \& Guariento, M. E. (2013). A satisfação com a vida e a funcionalidade em idosos atendidos em um ambulatório de geriatria. Ciência \& Saúde Coletiva, 18(12), 3475-3482.

Tomomitsu, V., Scandiuzzi, M. R., Perracini, M. R., \& Neri, A. L. (2014). Fatores associados à satisfação com a vida em idosos cuidadores e não cuidadores. Revista Ciência \& Saúde Coletiva, 19(8), 3429-3440.

Recebido em: 24/01/2018

Última Revisão: 22/10/2018

Aceite final: 13/11/2018 


\section{Sobre os autores:}

Daniel Vicentini de Oliveira: Doutor em Gerontologia pela Universidade Estadual de Campinas. Mestre em Promoção da Saúde pelo Centro Universitário de Maringá. Professor de Graduação e Pós-Graduação no Centro Universitário Metropolitano de Maringá. E-mail: d.vicentini@hotmail.com, Orcid: http://orcid.org/0000-0002-0272-9773

Anderson Rodrigues Brito: Especialista em Exercício Físico e Reabilitação do Idoso. Professor de Educação física. E-mail: andersonterco@hotmail.com, Orcid: http://orcid.org/0000-0003-3884-7752

Maria do Carmo Correia de Lima: Doutoranda em Gerontologia pela Universidade Estadual de Campinas. Mestre em Ciências da Saúde pela Universidade Federal de Pernambuco. E-mail: mariaclima@gmail.com, Orcid: http://orcid.org/0000-0001-9018-5325

Naelly Renata Saraiva Pivetta: Mestre em Gerontologia pela Universidade Estadual de Campinas. Fisioterapeuta. E-mail: naellypivetta@outlook.com, Orcid: http://orcid.org/0000-0002-2822-4618

José Roberto Andrade do Nascimento Júnior: Doutor em Educação física pela Universidade Estadual de Maringá. Mestre em Educação física pela Universidade Estadual de Maringá. Professor de graduação e pós-graduação na Universidade Federal do Vale do São Francisco E-mail: jroberto.jrs01@gmail.com, Orcid: http://orcid.org/0000-0003-3836-6967 
